\title{
Right-left prevalence in spatial compatibility
}

\author{
ROBERTO NICOLETTI \\ Università di Bologna, Bologna, Italy \\ and \\ CARLO UMILTÀ \\ Università di Parma, Parma, Italy
}

\begin{abstract}
Four experiments explored the effect of right/left cues on the spatial compatibility effects of the above/below and near/far dimensions. Experiment 1, in which stimuli and responses were positioned either above or below a central reference location, showed a strong spatial compatibility effect. Experiment 2 replicated Experiment 1 with the exception that right/left cues, incongruent with the above/below ones, were added. Compatibility proved to be stronger along the right/left dimension. In Experiment 3, near/far was the dimension according to which the cues were defined while the right/left dimension provided competing cues. Again, spatial compatibility was stronger along the right/left dimension. In Experiment 4, as in Experiment 2, the spatial cues involved the above/below and right/left dimensions. This time, the experimental procedure was such as to allow the competing spatial cues to vary independently and orthogonally. The results showed reliable compatibility effects for both dimensions, although they were apparently much stronger for the right/left one. Such findings are discussed with reference to the prevailing hypotheses proposed to explain S-R compatibility and the right/left confusion phenomenon observed in locational-discrimination tasks.
\end{abstract}

Among the factors that can influence speed of response in a choice reaction time (RT) task, stimulusresponse (S-R) compatibility is likely to be one of the most important (see, e.g., Teichner \& Krebs, 1974). Typically, response latencies are between 40 and $\mathbf{8 0 ~ m s e c ~ f a s t e r ~ f o r ~ c o m p a t i b l e ~ S - R ~ p a i r i n g s ~ t h a n ~ f o r ~}$ incompatible ones.

The notion of S-R compatibility originated with Fitts and Seeger (1953), who showed that responses were much faster when the mapping rules of the stimulus display corresponded to those of the response device than when they did not. Recently, Simon, Sly, and Vilapakkam (1981) aptly distinguished three types of compatibility: symbolic compatibility, spatial compatibility, and a third type of compatibility that can be termed the Simon effect (Hedge \& Marsh, 1975).

Symbolic compatibility results from the correspondence between the verbal labels associated with the stimulus and the response. For example, if there are red and green lights and red and green keys, re-

This research was supported by funds from the Consiglio Nazionale delle Ricerche and the Ministero della Pubblica Istruzione. The authors would like to thank Professors G. Berlucchi, C. A. Marzi, and G. Rizzolatti for helpful discussion of the experiments. They are also deeply indebted to Professor J. R. Simon for a critical reading of an earlier version of the manuscript. Requests for reprints should be sent to: Carlo Umilta, Istituto di Fisiologia Umana, Universita di Parma, Via A. Gramsci, 14,43100 Parma, Italy. Roberto Nicoletti is now at the Department of Psychology of the University of Padua, Padua, Italy. sponse latencies are shorter when the light stimulus indicates the use of the key defined by the same color than when it indicates the use of the other key (Hedge \& Marsh, 1975; Simon et al., 1981; Simon \& Sudalaimuthu, 1979).

Spatial compatibility is said to exist when the location of the stimulus indicates the location of the correct response and the two locations correspond. For example, response latencies are shorter when the right one of a pair of stimuli commands a response with the right one of a pair of keys than when it commands a response with the left key. Similarly, response latencies are shorter when the left stimulus requires a response with the left than when it requires a response with the right key (Anzola, Bertoloni, Buchtel, \& Rizzolatti, 1977; Brebner, Shepard, \& Cairney, 1972; Nicoletti, Anzola, Luppino, Rizzolatti, \& Umiltà, 1982; Simon, 1969).

The Simon effect can be observed when the position at which the stimulus appears and that at which the response takes place provide an irrelevant, but effective, cue (Craft \& Simon, 1970; Hedge \& Marsh, 1975; Simon, 1968; Simon, Hinrichs, \& Craft, 1970; Simon \& Rudell, 1967; Simon et al., 1981; Wallace, $1971,1972)$. For example, even if the relevant property of the S-R pairing is color, and thus the situation is paradigmatic of symbolic compatibility, response latencies are faster when the position of the stimulus and that of the response correspond (i.e., they are both on the right or left side of the body midline), than when they do not (i.e., the stimulus appears on 
one side while the corresponding response device is on the other side).

As for the stage of processing at which compatibility emerges, Teichner and Krebs (1974) suggested a translation stage which immediately precedes that of response selection. These authors distinguished four temporal components in visual choice reaction time: (1) a constant representing the sum of various neural transmission lags, (2) the time required for stimulus categorization, (3) the time required to perform some type of translation between stimulus and response, and (4) the time required for response selection. Accordingly, they ascribed compatibility effects to the third component and defined the degree of compatibility inherent in a task as "the proportion of choice reaction time that is attributable to stimulusresponse translation time" (Teichner \& Krebs, 1974, p. 91). This notion implies that compatibility must be dependent on the number of stimulus-response coding transformations: the fewer the translations required, the higher the degree of compatibility. Therefore, the highest level of compatibility is obtained when the same code is employed for both stimulus and response.

If we consider the three types of compatibility outlined above, it seems clear that an explanation in terms of S-R coding transformation is appropriate for symbolic compatibility. A task that lacks symbolic compatibility requires a translation from the verbal code describing the stimulus to the verbal code describing the response, a step that would not be required when the stimulus and the response shared the same code.

A similar interpretation for spatial compatibility and the Simon effect is less obvious. These types of compatibility have been hypothesized to result from a subject's tendency to respond in the direction of the source of stimulation (see Simon, 1968, and Simon \& Rudell, 1967, for the Simon effect; see Simon, 1969, for spatial compatibility) or from a correspondence between the spatial (or verbal) codes associated with the stimulus and the response (see Wallace, 1971, for the Simon effect; see Nicoletti et al., 1982, for spatial compatibility). According to the former hypothesis, relatively long response latencies are observed when the position of the stimulus does not match the place of the signaled response because an inappropriate tendency to respond toward the source of the stimulus must be inhibited. In terms of the Teichner and Krebs (1974) model, this hypothesis seems to suggest the stage of response selection as the locus where the compatibility effect occurs. In contrast, the code correspondence hypothesis fits very well with the notion that compatibility effects emerge at a processing stage in which the position code of the stimulus and that of the response are compared. When the two codes correspond (compatible S-R pairings), no translational activity is required, whereas when they do not correspond (incompatible $S-R$ pairings), some type of translation must take place. Actually, this explanation is identical to that given for symbolic compatibility, the only difference being in the nature of the codes. In the case of symbolic compatibility, they are very likely to be verbal, whereas in the case of both spatial compatibility and the Simon effect, they could be either spatial or verbal. In other words, it is not apparent whether the subjects make use of spatial codes or of the corresponding verbal descriptions in comparing the position of the stimulus with that of the response.

Recently, we (Nicoletti et al., 1982) have presented empirical evidence in favor of the code correspondence as an explanation of spatial compatibility. In that study, two light stimuli were shown in the same hemifield while the subject had either one hand on each side of the body midline or both hands on the same side of the midline. The spatial compatibility effects appeared to depend on the coding of the relative position of stimuli and responses, and not on a tendency to respond toward the source of stimulation. However, we were unable to determine the nature, spatial or verbal, of the codes. The present experiments addressed this issue.

Since the Nicoletti et al. study was limited to spatial compatibility, the present study was too. We believe that the term "spatial compatibility" must be restricted to those compatibility effects obtained when the position of the stimulus commands a determined response. This definition leaves aside the Simon effect, which is observed when the position of the stimulus and that of the response provide irrelevant locational cues (see Nicoletti et al., 1982; Simon et al., 1981).

Direct testing of the nature of the position codes involved in spatial compatibility is hardly feasible, but it seems that sound, though indirect, evidence could be gathered by utilizing the so-called right/left confusion effect whose verbal origin is well established. A number of recent studies have shown that it takes longer to make locational discriminations when the relevant spatial dimensions are described by the locative terms "right" and "left" than when they are described by the locative terms "above" and "below" (Farrell, 1979; Maki, Grandy, \& Hauge, 1979; Maki, Maki, \& Marsh, 1977; Sholl \& Egeth, 1981). Typically, the experimental task required judging the relative locations of two stimuli along either the horizontal dimension (i.e., right or left) or the vertical dimension (i.e., above or below). The subjects were consistently slower in the former than in the latter condition. This effect, often referred to as the right/left confusion, is usually attributed to the symmetry of the body and nervous sytem along the horizontal dimension and their asymmetry along the vertical dimension (see, e.g., Corballis \& Beale, 1976). It has also been shown that there is a continued growth of left-right discrimination ability in children from 5 through 10 years of age (Boone \& Prescott, 1968). 
Moreover, children learn other words associated with spatiality (such as up, down, high, low, middle, front, and back) much earlier and much more easily than right and left (Corballis \& Beale, 1976). Thus, the difficulty adults demonstrate in making right/left locational discriminations is likely to be a residual trace of the difficulty young children have in using the appropriate verbal labels for right and left positions. Accordingly, it is generally held (see Corballis \& Beale, 1976; Maki et al., 1979; Sholl \& Egeth, 1981; but see Farrell, 1979, for a different opinion) that applying verbal lables to right/left locations is the source of the effect, and Sholl and Egeth (1981), by disentangling the process of verbal encoding from other processes, have convincingly shown the former to be the determining factor in the right/left confusion.

Now, let us imagine a choice reaction time task in which the position of the stimulus is the only relevant cue for choosing the locus of the correct response (i.e., a task paradigmatic of spatial compatibility) and the relevant spatial relations can be described unambiguously in terms of either the above/below or the right/left dimension. In this situation, both pairs of locational cues would be perfectly predictive of the correct response. If the subjects used verbal codes to represent the spatial locations, a prevalence of the above/below dimension should be expected because the operation of verbal encoding is faster and simpler for that dimension than for the right/left one. In order to test this hypothesis, Experiment 1 attempted to demonstrate spatial compabitility effects when stimuli and responses were defined in terms of above and below positions and Experiment 2 was aimed at investigating the influence of redudant right/left cues on the spatial compatibility (if any) brought about by the mapping of stimuli and responses along the above/below dimension. It was assumed, in accordance with previous findings (Nicoletti et al., 1982), that spatial compatibility arises from a correspondence between the locational codes of stimulus and response, and it was predicted that, if those codes were verbal, no effect of the right/left cues on the above/below spatial compatibility would be observed.

\section{EXPERIMENT 1}

Experiment 1 was essentially a repetition of previous experiments that had shown the spatial compatibility effect for the right/left dimension. The only notable difference was that the light stimuli and the response keys were arranged vertically. Simon, Mewaldt, Acosta, and Hu (1976) have already demonstrated above/below compatibility effects, but that study dealt with symbolic compatibility and the Simon effect. In it, the subjects were faster when instructed to move a toggle switch up in response to a high-pitched tone and down in response to a low- pitched tone than they were when the instructions were for the opposite (symbolic compatibility), while the top or bottom position of the speaker yielded an irrelevant, but effective, spatial cue (Simon effect). Since the present study was concerned with spatial compatibility, we felt it appropriate to demonstrate that this type of compatibility also occurs in the above/below dimension.

\section{Method}

Subjects. Eight 19-27-year-old students at the University of Bologna served as paid subjects. They were all right-handed, had normal or corrected-to-normal visual acuity, and were naive as to the purpose of the experiment.

Apparatus. The experiment took place in a soundproof cubicle. The subject, with both eyes open, sat in front of a wooden panel on which two light-emitting-diodes (LEDs) were fastened. The head was positioned in an adjustable head-and-chin rest, so that the distance between the eyes and the center point of the panel was $100 \mathrm{~cm}$. The apparatus was indirectly illuminated from above, and the luminance of the ambient light was $6 \mathrm{~cd} / \mathrm{m}^{2}$. The fixation point consisted of a black patch subtending a visual angle of about $1 \mathrm{deg}^{2}$ in area and located in the center of the panel. The subject held with his/her hands two brass cylinders each with a push button on top. The two cylinders were placed on two adjacent vertical wooden shafts of different heights one positioned in front of the other. The distance between one shaft and the display panel was $83 \mathrm{~cm}$; the other shaft was $3 \mathrm{~cm}$ further away from the panel. One of the cylinders was $45 \mathrm{~cm}$ higher than the other, but both were exactly aligned with the midline of the subject's body and the midline of the display panel. A schematic drawing of the way the stimuli and responses were arranged is shown in Figure 1. The light stimuli (duration $100 \mathrm{msec}$ and luminance about $32 \mathrm{~cd} / \mathrm{m}^{2}$ ) subtended about $0.45 \mathrm{deg}$ of visual angle and were positioned $27 \mathrm{deg}$ exactly above or below the fixation mark. An acoustic warning signal preceded the light by an interval of $1 \mathrm{sec}$. From the warning signal to the execution of the response, the subject had to maintain his/her gaze on the fixation point, and eye position was continuously monitored by a television camera that allowed a deflection of about $1 \mathrm{deg}$ of visual angle to be reliably detected. If an eye movement was observed, that trial was discarded but not replaced. Eye movements were monitored, even though they could not have changed the relative positions of the stimuli, in order to control for possible retinal effects. The interstimulus interval was 5 sec. Interval timing was achieved with interval generators, and response

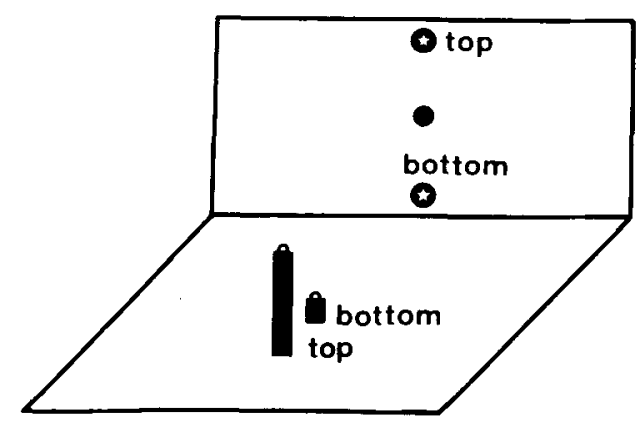

Stimuli on the vertical plane

Response-keys on the horizontal plane

Figure 1. Schematic diagram of stimulus display and response keys for Experiment 1. The two shafts with the response keys were actually aligned. In this drawing they are shown in perspective. 
latencies were recorded to the nearest millisecond by an electronic counter that was started with the onset of the light stimulus and stopped by the switch press.

Procedure. There were two experimental sessions on 2 consecutive days, preceded by an informal practice session. Trials were given in two blocks separated by a 2 -min rest period. During each block ( 80 trials), the two lights appeared above or below the fixation mark according to a quasi-random sequence that allowed a maximum of three consecutive presentations of the same (top or bottom) light. The subject was instructed to press one of the two buttons with his/her thumb as fast as possible following onset of a light. Each session comprised two conditions of S-R pairing. In the compatible condition, the subject was required to respond to the top light with the top hand and to the bottom light with the lower hand. In the incompatible condition, the assignment was reversed so that the top light corresponded to the bottom hand, and vice versa. The order of conditions was counterbalanced across sessions and subjects. In either the compatible or the incompatible condition, half of the responses were given with the right hand above the left hand and half were given with the opposite arrangement. For half of the subjects, the right hand was on the front cylinder and the left on the rear cylinder; for the other half, this arrangement was reversed. The instructions stressed both speed and accuracy. With the exception of the practice trials, no feedback was given to the subject about speed or accuracy. Errors were discarded but not replaced. The responses given after three consecutive presentations of the same light were not considered in the analysis because the fourth stimulus became perfectly predictable as a result of the type of quasi-random sequencing employed.

\section{Results and Discussion}

Errors, including eye movements, were extremely rare (less than $1 \%$ ) and were not submitted to statistical analysis. The correct mean RTs were entered in a four-way analysis of variance in which the withinsubjects factors were light position (top or bottom), hand position (top or bottom), and responding hand (right or left); hand arrangement (right or left hand in front) was a between-subjects factor. The bottom light was responded to faster than the top one [398 vs. $421 \mathrm{msec} ; F(1,7)=6.48$, $p<.05$ ] by six of the eight subjects. The interaction between light position and hand position was highly significant $[F(1,7)=$ $50.17, p<.001]$ and showed that the bottom hand was $66 \mathrm{msec}$ faster than the top hand in responding to the bottom light [365 vs. $431 \mathrm{msec} ; F(1,7)=74.50$, $\mathrm{p}<.001$, whereas the top hand was $69 \mathrm{msec}$ faster than the bottom hand in responding to the top light [387 vs. $456 \mathrm{msec} ; F(1,7)=33.25, p<.001]$. Every subject demonstrated this interaction. A summary of the results is given in Table 1 .

The main effect of light position was unexpected and was not confirmed by Experiment 4 . It might be attributed to the fact that the upper hemiretinae are more sensitive than the lower hemiretinae (see Haines \& Gilliland, 1973; Payne, 1967).

Of greater importance was the significant interaction, which showed a clear-cut spatial compatibility effect. This finding confirmed that spatial compatibility is not confined to the horizontal dimension but occurs in the vertical dimension as well. Such a result prompted a second experiment that was aimed at
Table 1

Mean Latencies (RTs in Milliseconds) in Experiment 1 as a Function of Stimulus Position (Top or Bottom), Response Position (Top or Bottom), and Responding Hand (Right or Left)

\begin{tabular}{lcccc}
\hline & \multicolumn{2}{c}{ Top Light } & & \multicolumn{2}{c}{ Bottom Light } \\
\cline { 2 - 3 } & Mean RT & SD & Mean RT & SD \\
\hline \multirow{4}{*}{ Right } & 377 & Top Hand & & \\
Left & 396 & 60 & 449 & 99 \\
& & 92 & 414 & 80 \\
Right & 457 & Bottom Hand & & \\
Left & 454 & 88 & 365 & 91 \\
\hline
\end{tabular}

determining the influence of right/left cues on the above/below spatial compatibility.

\section{EXPERIMENT 2}

Experiment 2 replicated Experiment 1, with the exception that both the light stimuli and the response devices were located either to the right or to the left of the body midline. Both lights and keys could still be differentiated in terms of the above/below dimension.

\section{Method}

Subjects. Eight new subjects selected as before took part in the experiment.

Apparatus. The apparatus was the one described for Experiment 1. The LEDs were 27 deg to the left or right and 27 deg above or below the fixation point. Correspondingly, the response devices were $22 \mathrm{~cm}$ to the left or right of the body midline and were positioned one above the other at a distance of $45 \mathrm{~cm}$. Hence, for both light stimuli and response devices there were four possible positions: top right, top left, bottom right, and bottom left. Figure 2 shows a schematic drawing of the experimental setup.

Procedure. The procedure exactly replicated that of Experiment 1 . The instructions were given in terms of top/bottom lights and top/bottom hands, and the experimenter purposely avoided referring to the right/left positions. However, in Experiment 2, the condition that was compatible for the above/below dimension was incompatible for the right/left dimension, whereas the condition that was incompatible for the above/below dimension was compatible for the right/left dimension. That is, in the compatible condition for the above/below dimension, responding hand and light stimulus were placed on opposite sides (i.e., right-left or leftright) in relation to the body midline. Conversely, in the incompatible condition for the above/below dimension, the position of light and response was congruent (i.e., right-right or left-left) in relation to the body midline. This was not true in Experiment 1, in which neither the lights nor the response devices were displaced horizontally. In each session, there were two blocks of $\mathbf{4 0}$ trials for each experimental condition (compatible or incompatible for above/below). For example, in the case of the compatible condition, in one block the subjects responded to the top light on the left with the top hand on the right and to the bottom light on the right with the bottom hand on the left. In the other block, they responded to the top light on the right with the top hand on the left and to the bottom light on the left with the bottom hand on the right. Therefore, responding hand and hand position were counterbalanced so that in half of the trials the right hand was above the left hand and in half it was below. Similarly, in half of the 
A

B

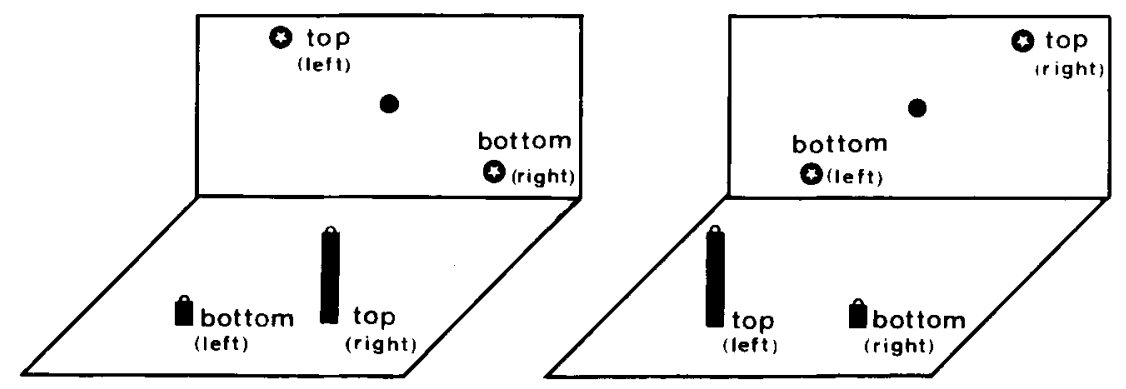

Stimuli on the vertical plane

Response-keys on the horizontal plane

Figure 2. Schematic diagrams of the two S-R arrangements of Experiment 2.

trials, the top light appeared in the right field and in the other half it appeared in the left field.

In brief, the main difference between Experiment 1 and Experiment 2 is that in the former the only valid cues to responding were the top and bottom positions of stimuli and responses, wherreas in the latter the right and left positions also were perfectly predictive of the correct response. In other words, in Experiment 2 the above/below and right/left cues were redundant and both could be effectively utilized for emitting a correct response. The instructions were given in terms of the above/below dimension in order to allow for a direct comparison with Experiment 1.

\section{Results}

There were virtually no errors in either condition (less than 1\%), and they were not analyzed. Correct RTs were submitted to a two-way within-subjects analysis of variance, with type of pairing (compatible for the above/below or the right/left dimension) and responding hand (right or left) as factors. A summary of the results is given in Table 2. Only type of pairing was significant $[\mathrm{F}(1,7)=17.81, \mathrm{p}<.005]$. RTs of the condition compatible for right/left were $57 \mathrm{msec}$ faster than those for the condition compatible for above/below (329 vs. $386 \mathrm{msec}$ ), and all the eight subjects showed the effect. In other words, for the above/below dimension, incompatible RTs were faster than compatible RTs, whereas for the right/left dimension, the opposite was true.

\section{Discussion}

The main finding of Experiment 2 was that right/ left cues could affect the above/below S-R pairings

Table 2

Mean Latencies (RTs in Milliseconds) in Experiment 2 as a Function of S-R Pairing (Compatible for Above/Below or Compatible for Right/Left) and Responding Hand (Right/Left)

\begin{tabular}{lccccc}
\hline & \multicolumn{2}{c}{$\begin{array}{c}\text { Compatible for } \\
\text { Above/Below }\end{array}$} & & \multicolumn{2}{c}{$\begin{array}{c}\text { Compatible for } \\
\text { Right/Left }\end{array}$} \\
\cline { 2 - 3 } \cline { 5 - 6 } & Mean RT & SD & & Mean RT & SD \\
\hline Right Hand & 383 & 27 & 327 & 49 \\
Left Hand & 389 & 29 & 331 & 44 \\
\hline
\end{tabular}

suggested by the instructions to the point of actually reversing the expected spatial compatibility effect. There are two possible explanations for this unexpected outcome. Both arise from the observation that the incompatible condition for above/below was, in fact, compatible for right/left, and vice-versa.

According to one explanation, the results actually showed a quite strong right/left spatial compatibility effect. It implies that the right/left cues were more salient than the above/below ones and thus the subjects chose to use the former for mapping the stimulus to the response. An alternative explanation suggests that the subjects always chose the cues that rendered the condition compatible. That is, they used a right/left S-R pairing when it was compatible and disregarded it in favor of an above/below pairing when the latter was the compatible one. If this was the case, then the results show that right/left spatial compatibility effects are stronger than above/below spatial compatibility effects. A direct test of these two interpretations was postponed to Experiment 4.

The main purpose of Experiment 2 was to clarify the nature of the codes involved in spatial compatibility. The results allow us to discard the possibility that verbal labels were used to describe the relative positions of stimuli and responses. As already noted, there is sound evidence that in locational discrimination tasks, the right/left confusion is a verbal labeling problem (Corballis \& Beale, 1976; Maki et al., 1979; Sholl \& Egeth, 1981). In our experiment, one would have predicted a completely different result if the subjects had used verbal labels. That is, the presence of right/left cues should have been immaterial for the processing of above/below cues, which, moreover, were the only cues mentioned in the instructions, or, alternatively, above/below compatibility should have been, if anything, stronger than right/left compatibility.

A way of reconciling the findings of Experiment 2 with the right/left confusion notion can be found if one attributes spatial compatibility and the right/left 
confusion to two different types of codes, both associated with positions in space of stimuli and responses. The right/left confusion could depend on the use of verbal codes, whereas spatial compatibility could depend on the use of spatial codes.

Before proceeding with an empirical test of the two hypotheses advanced to explain the results of Experiment 2, a further interpretation must be considered. It could simply take longer to locate positions within the vertical than within the horizontal dimension, and this would explain the prevalence of right/left over above/below cues. Although such a possibility seemed unlikely in view of the results of previous studies (Maki et al., 1979; Sholl \& Egeth, 1981), we decided to test it in Experiment 3.

\section{EXPERIMENT 3}

In Experiment 2, the two pairs of locational cues involved two orthogonal spatial dimensions. Thus, there need not be any peculiarity about right and left locations and the results could be attributable to a prevalence of horizontal over vertical S-R pairings. In order to test this possibility, in Experiment 3 we tried to replicate the results of Experiment 2 by employing the horizontal dimension for both S-R pairings. The locative terms mentioned in the instructions were near and far, while the conflicting cues were again right and left. In all other respects, Experiment 3 was very similar to Experiment 2.

\section{Method}

Subjects. Eight new subjects, selected as before, took part in the experiment.

Apparatus. The apparatus was the one described for Experiment 1 . The LEDs were horizontally displaced so that the near one was $4 \mathrm{deg}$ and the far one $27 \mathrm{deg}$ from the fixation point. Correspondingly, the response devices were placed 7 and $30 \mathrm{~cm}$ from the body midline. With reference to the midsagittal plane, both the light stimuli and the response devices could be on either the left or right side. Thus, there were four possible positions: near right, near left, far right, and far left. A schematic representation of the arrangement of the stimuli and response devices is shown in Figure 3.

Procedure. The procedure exactly replicated that described for Experiment 2. The instructions were given in terms of near/far lights and near/far hands. In the compatible condition, the subject had to respond to the far light with the far hand and to the near light with the near hand; the incompatible condition required a response with the near hand to the far light, and vice versa. In the instructions, no mention was made of the right/left dimension. However, it must also be pointed out that in the present experiment the two pairs of locational cues were redundant; that is, both could be used to yield correct $S-R$ pairings. The experimental situation was such that the condition that was compatible for the near/ far dimension was incompatible for the right/left dimension, whereas incompatibility for the near/far dimension implied compatibility for the right/left dimension. In other words, when the near hand responded to the near light and the far hand to the far light, the responding hand and the stimulus were on opposite sides (i.e., right-left or left-right) in relation to the midline. Conversely, the responding hand and the light were on the same side (i.e., right-right or left-left) when the requirement was to respond with the far hand to the near light, and vice versa.

Also, in this experiment there were two blocks of 40 trials for each condition (compatible or incompatible for near/far) in each session. For example, when the condition was compatible, in one block, the subject responded to the near light on the right with the near hand on the left and to the far light on the left with the far hand on the right; in the other block, he or she responded to the near light on the left with the near hand on the right and to the far light on the right with the far hand on the left. Therefore, in either the compatible or the incompatible condition, half of the responses were given with the right hand nearer than the left and half were given with the opposite arrangement. Similarly, in half of the trials, the near light appeared to the right of fixation and in the other half it appeared to the left.

\section{Results}

As usual, the few errors observed (less that 1\%) were not analyzed. A two-way within-subjects analysis of variance was conducted on correct RTs, with type of pairing (compatible for the near/far or the right/left dimension) and responding hand (right or left) as factors. The results are summarized in Table 3.

There was a highly significant main effect of type of pairing $[\mathrm{F}(1,7)=98.77, \mathrm{p}<.001]$, which showed that RTs compatible for right/left were $86 \mathrm{msec}$

A

B

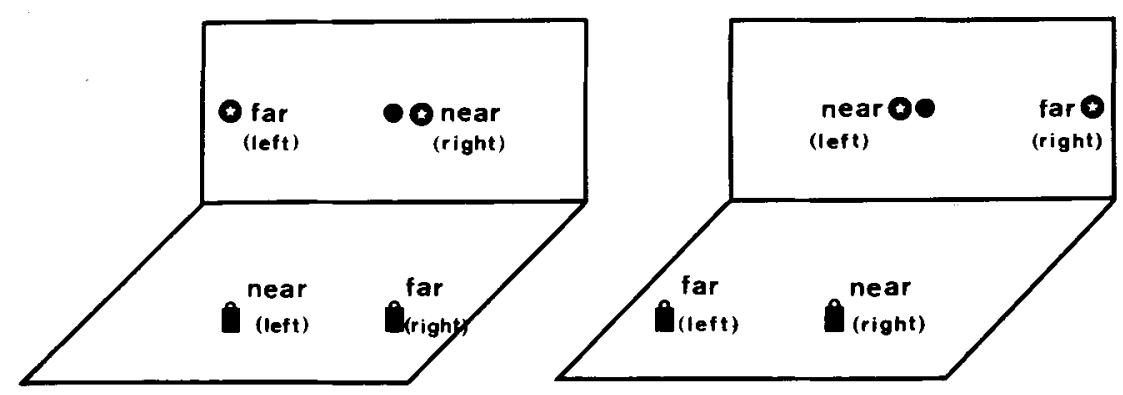

Stimuli on the vertical plane

Response-keys on the horizontal plane

Figure 3. Schematic diagrams of the two S-R arrangements of Experiment 3. 
Table 3

Mean Latencies (RTs in Milliseconds) in Experiment 3 as a Function of S-R Pairing (Compatible for Near/Far or Compatible for Right/Left) and Responding Hand (Right/Left)

\begin{tabular}{cccccc} 
& \multicolumn{2}{c}{$\begin{array}{c}\text { Compatible for } \\
\text { Near/Far }\end{array}$} & & \multicolumn{2}{c}{$\begin{array}{c}\text { Compatible for } \\
\text { Right/Left }\end{array}$} \\
& Mean RT & SD & & Mean RT & SD \\
\hline Right Hand & 392 & 32 & & 285 & 35 \\
Left Hand & 384 & 42 & & 320 & 41 \\
\hline
\end{tabular}

faster than those compatible for near/far (302 vs. $388 \mathrm{msec}$ ). All eight subjects showed the effect. In other words, the near/far dimension yielded incompatible RTs faster than it yielded compatible ones, whereas the right/left dimension yielded the opposite outcome. Contrary to expectations, the main effect of responding hand and the interaction between type of pairing and responding hand were also significant $[F(1,7)=9.31, p<.05$, and $F(1,7)=14.62, p<.01$, respectively]. The right hand was $14 \mathrm{msec}$ faster than the left, particularly so in the condition compatible for the right/left dimension [ 285 vs. $320 \mathrm{msec} ; F(1,7)$ $=32.69, \mathrm{p}<.001]$. However, a nonsignificant advantage for the left hand was observed in the condition compatible for the near/far dimension (384 vs. $392 \mathrm{msec}$ ). These last two findings should not be given too much weight since they were not replicated in either Experiment 2 or Experiment 4. An explanation for them might be found in the fact that for right-handers right is more salient than left (Olson \& Laxar, 1973) and the effect could manifest itself only when both S-R pairings concerned the horizontal dimension.

\section{Discussion}

The results of Experiment 3 confirmed those of Experiment 2 in showing the effectiveness of right/ left cues in counteracting the S-R pairings suggested by the instructions. Also, in this case the two tentative explanations put forward to interpret a similar outcome for Experiment 2 are equally tenable. It could be that the subjects considered only the right/ left cues, in which case the results indicate a strong effect of compatibility along the right/left dimension. Alternatively, it could be that they selected the S-R pairing that was compatible in a given experimental condition, in which case the results indicate that spatial compatibility effects are stronger in the right/left than in the near/far dimension.

In the present experiment, however, the hypothesis of a prevalence of the horizontal over the vertical dimension can be rejected because the two conflicting S-R pairings were mapped along the same, horizontal, dimension.

It seems that there is something special about right and left locations that renders them more salient than other locations, such as above, below, near, and far. Since verbal labeling is considered to be the cause of the slower processing of right and left locations in locational judgments, the right/left prevalence observed in spatial compatibility cannot be attributed to the use of verbal codes in the S-R translation process: Nonverbal codes must be involved in bringing about spatial compatibility effects. Hence, the locus at which both spatial compatibility and the prevalence of right/left S-R pairings occur is likely to be a stage where the relative locations of stimuli and response are compared on the basis of spatial codes.

In the two previous experiments, the right/left and above/below or near/far dimensions were not varied orthogonally. The two possible ways of encoding S-R pairings excluded one another, so that, in a given experimental trial, only one could be used. Such procedure, while allowing the observation of the right/left prevalence, could not clarify whether the subjects considered solely the right/left cues or those cues that were compatible. In Experiment 4, we addressed this issue by combining orthogonally the two dimensions. If the S-R pairings were based exclusively on the right/left cues, then only right/left spatial compatibility effects would be obtained, whereas, if both types of cues could be used in mapping stimuli to responses, then two types of spatial compatibility effects, one for each dimension, would be obtained. In the latter case, there is the additional prediction of stronger compatibility effects for the right/left dimension.

\section{EXPERIMENT 4}

The above/below dimension was chosen as the one mentioned in the instructions, and the right/left dimension once again provided the other locational cues. This time, however, the experimental situation was such as to allow the two dimensions to vary independently and orthogonally. In all other respects, Experiment 4 replicated Experiment 2.

\section{Method}

Subjects. Eight new subjects, selected as before, participated in the experiment.

Apparatus. The apparatus and the arrangement of the stimuli and the response devices were identical to those described for Experiment 2. A schematic representation of the experimental setup is shown in Figure 4.

Procedure. Except for the difference noted below, the procedure was the same as that in Experiment 2. Again, the instructions were given by mentioning only the above/below dimension and the experimenter purposely avoided any mention of the right/left dimension. However, there was one important difference in the procedure which allowed an orthogonal manipulation of the two compering dimensions. There were four experimental conditions instead of two as in Experiment 2. In the first condition, the S-R pairings were compatible for both the above/below and the right/ left dimension. For example, when the top right light was shown, the subject responded with the top right hand. The second condition comprised S-R pairings that were compatible only for the 
A

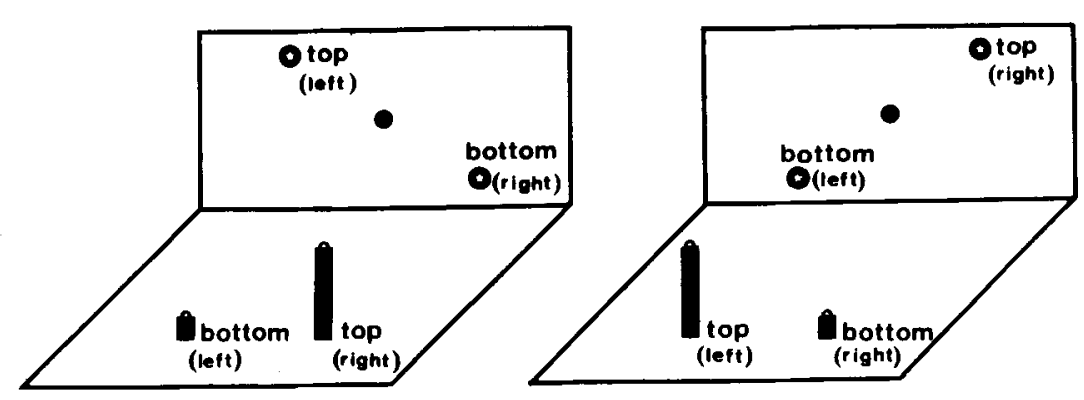

C

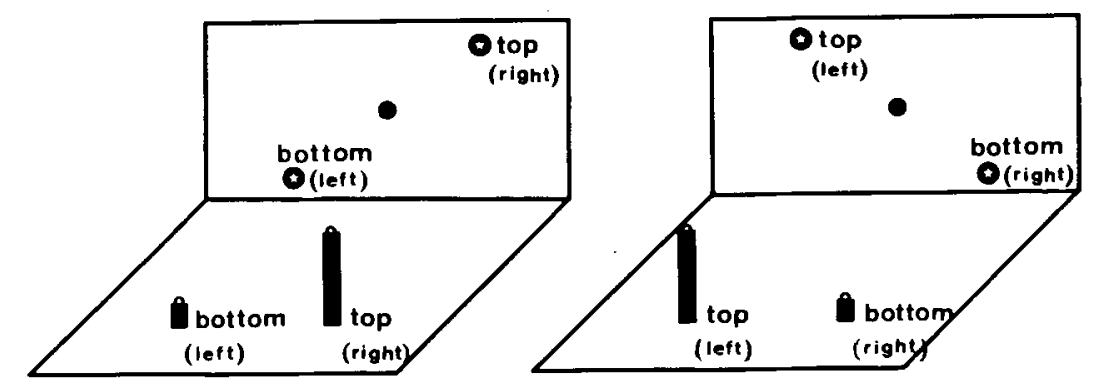

Stimuli on the vertical plane

Response-keys on the horizontal plane

B

D

Figure 4. Schematic diagrams of the four S-R arrangements of Experiment 4.

right/left dimension. For example, the top right light commanded a response with the bottom right hand. The third condition comprised S-R pairings compatible only for the above/below dimension. In this case, the top right light required a response with the top left hand. Finally, the fourth condition was compatible for neither dimension. For example, the top right light required a response with the bottom left hand. It must be noted that the second and the third conditions were those already employed in Experiment 2 . In each of the two sessions, the subjects were given eight blocks of $\mathbf{4 0}$ trials, two blocks for each experimental condition. The assignment of the right or left hand to the top or bottom position and of the top or bottom light to the right or left field was counterbalanced within subjects and across blocks, so that half of the trials were with the right hand above the left and the other half were with the opposite arrangement. Correspondingly, half of the trials were with the top light in the right field and half were with the bottom light in the right field. In the first condition, for example, one block required responding to the top light on the right with the top hand on the right and to the bottom light on the left with the bottom hand on the left. The other block required responding to the top light on the left with the top hand on the left and to the bottom light on the right with the bottom hand on the right.

\section{Results}

The errors (less than 1\%) were not analyzed. The correct RTs were submitted to a four-way withinsubjects analysis of variance with the following factors: position of the light in relation to the vertical dimension (top and bottom), position of the light in relation to the horizontal dimension (right and left), position of the hand in relation to the vertical dimen- sion (top and bottom), and position of the hand in relation to the horizontal dimension (right and left).

A summary of the results is given in Table 4. There were two highly significant first-order interactions, that is, the interaction between vertical arrangement of the light and vertical arrangement of the response $[F(1,7)=14.93, p<.01]$ and that between horizontal arrangement of the stimulus and horizontal arrangement of the response $[F(1,7)=$ $92.70, p<.001]$. No other source was significant. The top hand was $16 \mathrm{msec}$ faster than the bottom hand in responding to the top light [ $333 \mathrm{vs} .349 \mathrm{msec}$, $F(1,7)=20.02, p<.005]$. Correspondingly, the bottom hand was $18 \mathrm{msec}$ faster than the top hand in responding to the bottom light (331 vs. $349 \mathrm{msec}$, $F(1,7)=6.27, p<.05]$. Seven of the eight subjects showed this interaction. As for the other dimension, the right hand was $63 \mathrm{msec}$ faster than the left in responding to the right light [306 vs. $369 \mathrm{msec}, F(1,7)$ $=52.18, \mathrm{p}<.001]$. Similarly, the left hand was $62 \mathrm{msec}$ faster than the right hand in responding to the left light $[312$ vs. 374 msec, $F(1,7)=135.39, p<$ $.001]$. All eight subjects showed this interaction.

These results also can be described by making reference to the above-mentioned four experimental conditions. The fastest RTs were observed when the S-R pairing was compatible for both dimensions (303 $\mathrm{msec}$ ), and the slowest RTs were those for the 
Table 4

Mean Latencies (RTs in Milliseconds) in Experiment 4 as a Function of Stimulus Position (Top or Bottom and Right or Left), Response Position (Top or Bottom and Right or Left), and Responding Hand (Right or Left)

\begin{tabular}{|c|c|c|c|c|c|c|c|c|}
\hline & \multicolumn{4}{|c|}{ Right Light } & \multicolumn{4}{|c|}{ Left Light } \\
\hline & \multicolumn{2}{|c|}{ Top } & \multicolumn{2}{|c|}{ Bottom } & \multicolumn{2}{|c|}{ Top } & \multicolumn{2}{|c|}{ Bottom } \\
\hline & Mean RT & SD & Mean RT & SD & Mean RT & SD & Mean RT & SD \\
\hline \multicolumn{9}{|c|}{ Right Hand } \\
\hline $\begin{array}{l}\text { Top } \\
\text { Bottom }\end{array}$ & $\begin{array}{l}281 \\
313\end{array}$ & $\begin{array}{l}38 \\
66\end{array}$ & $\begin{array}{l}317 \\
314\end{array}$ & $\begin{array}{l}40 \\
44\end{array}$ & $\begin{array}{l}375 \\
392\end{array}$ & $\begin{array}{l}48 \\
33\end{array}$ & $\begin{array}{l}374 \\
354\end{array}$ & $\begin{array}{l}66 \\
47\end{array}$ \\
\hline \multicolumn{9}{|c|}{ Left Hand } \\
\hline $\begin{array}{l}\text { Top } \\
\text { Bottom }\end{array}$ & $\begin{array}{l}356 \\
366\end{array}$ & $\begin{array}{l}58 \\
65\end{array}$ & $\begin{array}{l}397 \\
358\end{array}$ & $\begin{array}{l}56 \\
32\end{array}$ & $\begin{array}{l}319 \\
321\end{array}$ & $\begin{array}{l}47 \\
43\end{array}$ & $\begin{array}{l}308 \\
299\end{array}$ & $\begin{array}{l}60 \\
45\end{array}$ \\
\hline
\end{tabular}

condition in which neither dimension yielded compatible S-R pairings $(383 \mathrm{msec})$. The other two conditions, which were compatible for only one dimension, gave intermediate results: $316 \mathrm{msec}$ when the right/left dimension was compatible and $360 \mathrm{msec}$ when the above/below dimension was compatible.

Overall, the compatibility effect for the right/left dimension was significantly larger than that for the above/below dimension $[62$ vs. $17 \mathrm{msec}, F(1,7)=$ $34.03, \mathrm{p}<.001]$. This was true for every subject.

\section{Discussion}

The results of Experiment 4 were clear in showing spatial compatibility effects due to both types of S-R pairings. It appears that the subjects could utilize two different types of spatial correspondence in mapping stimulus to response. Not surprisingly, in view of the results of Experiment 2, the right/left correspondence proved more effective than the above/below correspondence, even though the instructions were couched in terms of the latter.

The finding that the position of the light and that of the response undergo a two-way encoding process and that both of the ensuing codes are utilized in selecting the correct response allows us to reject one of the two interpretations proposed for the results of Experiments 2 and 3. One of them implied that only a single spatial dimension could be processed in a given trial and that the right/left dimeusion was the preferred one. The results of Experiment 4 showed that this was not the case. As predicted by the other hypothesis, the subjects can process the two dimensions simultaneously and spatial compatibilities along both dimensions add their effects in determining the speed of a correct response. The only apparent difference between the two dimensions is to be found in the stronger spatial compatibility yielded by right/left locational cues.

A point that must be discussed is the apparent decrease of the magnitude of the above/below spatial compatibility from Experiment 1 to Experiment 4. The above/below dimension, when processed in isolation, yielded an effect of compatibility that was much larger in Experiment 1 than in Experiment 4, in which the right/left dimension was also present [68 vs. 17 msec, $F(1,14)=10.15, p<.01]$. Also, Farrell (1979) found an interaction between right/left and above/below dimensions when subjects judged the direction of arrows that pointed up, down, left, or right and could appear above, below, to the left, or to the right of fixation. However, the effect there was just the opposite of that obtained here. In Farrell's study, above/below cues interfered with right/left discriminations, whereas right/left cues had no effect on up/down discriminations. This discrepancy gives support to the notion that right/left effects observed in spatial compatibility tasks and in locational discrimination tasks are due to different processing modes.

Another consideration confirms the spatial nature of codes that define the relative positions of stimulus and response and are involved in the translational process. It would seem that the strategy of describing the S-R pairings with four verbal labels (two for the light and two for the response) is much too complex to be utilized in a speeded task.

\section{CONCLUSION}

The above series of experiments was motivated by Simon et al. (1981), who differentiated three types of compatibility (i.e., symbolic compatibility, spatial compatibility, and the Simon effect), and by Nicoletti et al. (1982), who attributed one type of compatibility (i.e., spatial compatibility) to the correspondence, or lack of it, of the locational codes associated with stimuli and responses. This latter view is in accordance with a suggestion by Teichner and Krebs (1974), who ascribed spatial compatibility to a translation process of the S-R codes.

The main point at issue in the present study was the nature, verbal or spatial, of the codes involved in such a translation process and, by implication, in spatial compatibility. After showing spatial compatibility effects along the above/below dimension, we presented the subjects with stimuli and responses whose positions could be described by two pairs of spatial cues, that is, right/left and above/below or 
near/far. The two pairs of cues were redundant in the sense that each was perfectly predictive of the correct response. It was reasoned that if the codes on which spatial compatibility depends were verbal, the right/left dimension would have caused some difficulty to the subjects due to the right/left confusion effect.

The results of Experiments 2 and 4 showed that: (1) the subjects could utilize simultaneously both dimensions in mapping stimuli to responses, (2) both dimensions yielded reliable spatial compatibility effects, and (3) spatial compatibility effects were much stronger along the right/left dimension. Experiment 3 showed that the prevalence of right/left compatibility was dependent on the right and left locations and could not be attributed to a saliency of the horizontal dimension as compared with the vertical dimension. Apparently, there is something peculiar about the right and left locations that renders S-R pairings based on them predominant over S$R$ pairings based on other locations. This finding allows us to reject the hypothesis that the codes involved in spatial compatibility are verbal, that is, that they correspond to the verbal labels describing the relevant positions of stimuli and responses. Such codes must be nonverbal and are likely to be spatial.

The view that a position in space can be described in both verbal and spatial terms is rather intuitive and permits a reconciliation between the apparent contradiction between the right/left confusion effect observed in locational discrimination tasks and the right/left prevalence found in spatial compatibility tasks. The former would be caused by the use of verbal codes, whereas the latter would depend on the use of spatial codes. However, at this point, it becomes necessary to explain why right and left locations are more difficult to process when coded verbally and easier to process when coded spatially. As already noted, the most likely explanation of the verbal right/left difficulty is to be found in the symmetrical right-to-left axis of the human body (Corballis \& Beale, 1976), which causes the absence of a consistent natural referent in the horizontal dimension. Hence, subjects would find it difficult to apply arbitrary verbal labels for distinguishing between symmetrical right/left locations. In other words, locatives function to describe locations with respect to a particular point of reference and "right"/ "left" labels are difficult to use since the lack of such a reference point renders them ambiguous and ill-defined (see Maki et al., 1979). On the other hand, the prevalence of right/left S-R pairings in spatial compatibility tasks could be explained in terms of the notion of a "midline barrier" proposed by Bruner (1971) to describe the difficulty that young infants have in reaching across the midline. In accordance with this notion, Provine and Westerman (1979) have shown that, in infants of 9 to 20 weeks of age, visually directed hand extension and reaching progress from the ipsilateral domain to the midline and later to the contralateral domain. Thus, it seems that ipsilateral S-R links are present before contralateral ones. This being the case, it is not surprising that even in the adult S-R pairings on the same side of the body midline are predominant over S-R pairings that cross the body midline.

\section{REFERENCES}

Anzola, G. P., Bertoloni, G., Buchtel, H. A., \& Rizzolatti, G. (1977). Spatial compatibility and anatomical factors in simple and choice reaction time. Neuropsychologia, 15, 295-302.

Boone, D. R., \& Prescotr, T. E. (1968). Development of leftright discrimination in normal children. Perceptual and Motor Skills, 26, 267-274.

Brebner, J., Shepard, M., \& Cainney, P. (1972). Spatial relationships and S-R compatibility. Acta Psychologica, 36, 1-15.

Bruner, J. S. (1971). The growth and structure of skill. In K. J. Conolly (Ed.), Motor skills in infancy. New York: Academic Press.

Conballis, M. C., \& Beale, I. L. (1976). The psychology of left and right. Hillsdale, $\mathrm{NJ}$ : Erlbaum.

Craft, J. L., \& Simon, J. R. (1970). Processing symbolic information from a visual display: Interference from an irrelevant directional cue. Journal of Experimental Psychology, 83, 415-420.

FARrell, W. S. (1979). Coding left and right. Journal of Experimental Psychology: Human Perception and Performance, 5, 42-51.

FitTs, P. M., \& Seegen, C. M. (1953). S-R compatibility: Spatial characteristics of stimulus and response codes. Journal of Experimental Psychology, 46, 199-210.

Haines, R. F., \& Gilliland, K. (1973). Response time in the full visual field. Journal of Applied Psychology, 58, 289-295.

Hedge, A., \& Marsh, N. W. A. (1975). The effect of irrelevant spatial correspondence on two-choice response-time. Acta Psychologica, 39, 427-439.

Maki, R. H., Grandy, C. A., \& Hauge, G. (1979). Why is telling right from left more difficult than telling above from below. Journal of Experimental Psychology: Human Perception and Performance, 5, 52-67.

Maki, R. H., Maki, W. S., \& Marsh, L. G. (1977). Processing locational and orientational information. Memory \& Cognition. S, 602-616.

Nicoletti, R., Anzola, G. P., Luppino, G., Rizzolatti, G., \& Umiltà, C. (1982). Spatial compatibility effects on the same side of the body midline. Journal of Experimental Psychology: Human Perception and Performance, 8, 664-673.

Olson, G. M., \& LAXAR, K. (1973). Asymmetries in processing the terms "right" and "left." Journal of Experimental Psychology, 100, 284-290.

PAYNe, W. H. (1967). Visual reaction times on a circle about the fovea. Science, 155, 481-482.

Provine, R. R., \& Wegterman, J. A. (1979). Crossing the midline: Limits of early eye-hand behavior. Child Development, 50, 437-441.

Shold, M. J., \& Egeth, H. E. (1981). Right-left confusion in the adult: A verbal labeling effect. Memory \& Cognition, 9, 339-350.

Simon, J. R. (1968). Effect of ear stimulated on reaction time and movement time. Journal of Experimental Psychology, 78, 344-346.

Simon, J. R. (1969). Reactions toward the source of stimulation. Journal of Experimental Psychology, 81, 174-176.

Simon, J. R., Hinrichs, J. V., \& Craft, J. L. (1970). Auditory 
S-R compatibility: Reaction time as a function of ear-hand correspondence and ear-response-location correspondence. Journal of Experimental Psychology, 86, 97-102.

Simon, J. R., Mewaldt, S. P., Acosta, E., JR., \& Hu, J.-M. (1976). Processing auditory information: Interaction of two population stereotypes. Journal of Applied Psychology, 60, 354-358.

Simon, J. R., \& Rudell, A. P. (1967). The effect of an irrelevant cue on information processing. Journal of Applied Psychology, 51, 300-304.

Simon, J. R., Sly, P. E., \& Vilapakkam, S. (1981). Effect of compatibility of S-R mapping on reactions toward the stimulus source. Acta Psychologica, 47, 63-81.

Simon, J. R., \& Sudalaimuthu, P. (1979). Effects of S-R map- ping and response modality on performance in a Stroop task. Journal of Experimental Psychology: Human Perception and Performance, 5, 176-187.

Teichnen, W. H., \& Krebs, M. J. (1974). Laws of visual choice reaction time. Psychological Review, 81, 75-98.

WALLACE, R. J. (1971). S-R compatibility and the idea of a response code. Journal of Experimental Psychology, 88, 354-360.

WALlace, R. J. (1972). Spatial S-R compatibility effects involving kinesthetic cues. Journal of Experimental Psychology, 93, 163-168.

(Manuscript received June 14, 1983;

revision accepted for publication February 6, 1984.) 